\title{
Autorinnen und Autoren des Heftes
}

\author{
Hartmut Aden \\ geb. 1964, Rechtsreferendar, Doktorand an der Univer- \\ sität Hannover, Sempcrplatz. 1, 22303 Hamburg \\ Maren Brandenburger \\ gcb. 1968, MA, Gastdozentin an Zivildienstschulen, \\ Doktorandin an der Universität Hannover, Mainzerstr. \\ 132, 53179 Bonn \\ Johannes Caspar \\ geb. 1962, Dr. jur., Wissenschaftlicher Referent, For- \\ schungsstelle Umweltrecht, Fachbercich Rechtswissen- \\ schaft II, Universität Hamburg, Edmund Sicmers Allec \\ 1, 20146 Hamburg \\ Peter Derleder \\ Monika Frommel \\ geb. 1940, Dr. jur., Professor für Zivilrecht an der Uni- \\ versität Bremen, Fitgerstr. 15, 28209 Bremen \\ geb. 1946, Dr. jur., Professorin für Strafrecht und Kri- \\ minologic an der Christian Albrecht Universität Kiel, \\ Leibnizstr. 6, 24118 Kiel \\ Jürgen Habermas \\ geb. 1929, Dr. phil., em. Professor für Philosophic an \\ der Universität Frankfurt/M, Ringstr. 8 b, 825 19 Starn- \\ berg \\ Andreas Keller \\ geb. 1965 , Dipl. Pol., wissenschaftlicher Mitarbeiter am \\ Institut für Politikwissenschaft der Universität Mar- \\ burg, Posenerstr. 6, 35043 Marburg \\ Joachim Perels \\ geb. 1942, Dr. jur., Professor für Politische Wissen- \\ schaft an der Universität Hannover, Kantstr. 4, 30625 \\ Hannover \\ Wanja Andreas Welke \\ geb. 1971, Student der Rechtswisscnschaft an der Uni- \\ versitäe Frankfurt/M, Franz Rücker Allee, 42, 60487 \\ Frankfurt/M. \\ Sonja Wölte \\ geb. 1968, Studentin der Rechtswissenschaft an der \\ Universität Frankfurt/M, Schleusenstr. 15, 60327 \\ Frankfurt/M.
}

